\title{
Article \\ Study on the Performance of Liquid Metal Lubricated V-Groove Bearing Considering Turbulence
}

\author{
Mingyu $\mathrm{Xu}$ and Wei Chen *
}

check for

updates

Citation: Xu, M.; Chen, W. Study on the Performance of Liquid Metal Lubricated V-Groove Bearing Considering Turbulence. Appl. Sci. 2021, 11, 940. https://doi.org/ 10.3390/app11030940

Received: 10 December 2020 Accepted: 12 January 2021 Published: 21 January 2021

Publisher's Note: MDPI stays neutral with regard to jurisdictional clai$\mathrm{ms}$ in published maps and institutional affiliations.

Copyright: (C) 2021 by the authors. Licensee MDPI, Basel, Switzerland. This article is an open access article distributed under the terms and conditions of the Creative Commons Attribution (CC BY) license (https:// creativecommons.org/licenses/by/ $4.0 /)$.
Key Laboratory of Education Ministry for Modern Design and Roter-Bearing System, Xi'an Jiaotong University, Xi'an 710049, China; xumingyu@stu.xjtu.edu.cn

* Correspondence: chenw@mail.xjtu.edu.cn

\begin{abstract}
To study the application of liquid metal (LM) in the field of practical lubrication, the viscosity of gallium based liquid metal (GBLM) was measured initially, and the relationship between viscosity and temperature was fitted to obtain the viscosity under high temperature. Under the simulated high temperature and vacuum working environment of computed tomography tube (CTT) machines, considering the influence of turbulence, the changes, with eccentricity, of bearing capacity, discharge, friction power consumption, temperature rise, stiffness, and critical mass of the GBLM lubricated V-groove bearing (V-g B) were analyzed. Due to the special structure of V-g $\mathrm{B}$, the coordinate transformation was carried out and the turbulent Reynolds equation was solved by using the finite difference method and the local integral method. The bearing film thickness and pressure distribution under the two coordinate systems were analyzed and compared and the pressure distribution of V-g B under small eccentricity and large eccentricity was studied, respectively. The performance of GBLM lubricated V-g B was studied, which provides theoretical guidance and an analytical method for LM bearing of high-performance CT equipment.
\end{abstract}

Keywords: liquid metal; V-groove bearing; turbulence; lubrication; static and dynamic performance

\section{Introduction}

Computed tomography (CT) equipment can find the internal problems of the object before the implementation of measures. It can reduce the cost and risk, and plays an increasingly important role in medical diagnosis, equipment diagnosis, and other aspects. As the high-value and consumable parts of CT machines, one or two CTT are generally needed to be replaced once a year per CT equipment. Therefore, to medical giants in various countries, CTT is the core component in the development and production process of CT machines [1] and has a great market value.

In order to avoid the local damage of a fixed anode due to heat accumulation in the CTT, a mature technology is to use a rotating anode instead of a fixed one, so that the heat will be dispersed into an arc area, and not a point, preventing materials from local damage. Considering the actual working conditions of CTT, it is required that the lubricant used in the rotating anode should not pollute the vacuum tube. The lubricant should also be able to withstand high temperature and conduct electricity. Meanwhile, it is better to conduct heat. Gallium based liquid metal (GBLM) has excellent electric and thermal conductivity as well as fluidity. These qualities make it the best choice to lubricate the rotating anode. In the Netherlands, the Philips Medical Institution has produced the IMRC CTT [1]. This CTT changed the anode applied to medical diagnosis from fixed to rotating, and used a spiral groove anode bearing with LM as the lubricant. This technology has not only improved the performance of CTT, but also broadened the research field. Next, the technology was developed, comparatively well-established in Europe and America [2-5]. Professor Chen Wei of Xi'an Jiaotong University in China [6] provided a detailed design and research on this type of LM lubricated spiral groove bearing. 
GBLM has the advantages of low vapor pressure, high thermal conductivity, and good fluidity, but is also non-toxic and stable in thermo-physical properties. The research on GBLM is increasing, especially its lubrication performance, and its application is growing extensively [7]. For example, Hughes [8] used LM as a high temperature conductive lubricating fluid in magnetohydrodynamic bearings for nuclear power, and found that the bearings' capacity had been greatly improved; Burton [9] used gallium-indium-tin liquid metal (G-I-T LM) to lubricate and found that the resistance and calorific value of the brush with high current density decreased; Cerkema [10] discovered that GBLM could lubricate deep groove ball bearings in an oxygen free environment, at the same time, the surface tension and adhesion of GBLM had a great influence on the lubrication performance; Kezik [11] studied the lubrication properties of LM at different temperatures; Tian Yu's research [12] showed that GBLM could effectively prevent the welding of sliding interface under extreme pressure and high load; and Liu Weimin's team [13-16] has conducted many friction and wear tests, showing that a GBLM oxide film has a certain lubricating effect.

The research of spiral groove bearing has a history of more than 90 years [17]. Due to the spiral groove bearing's self-sealing performance, this kind of bearing does not need external oil supply equipment. The hydrodynamic sliding bearing realizes load carrying through the dynamic pressure oil film. Due to the special structure of the spiral groove bearing, this kind of bearing forms an oil film by the pumping pressure effect and periodic step effect of the spiral groove. Compared with the traditional ball bearing used in CTT, the spiral groove bearing has many advantages such as compact structure, good stability, low vibration noise, low friction power consumption, low wear, and long service life [17]. Therefore, it can better meet the needs of high-speed and stable rotation of the anode target.

At present, the research on the lubrication performance of GBLM is mainly focused on the effective anti-friction and load-bearing performances through the friction and wear tests. Meanwhile the theoretical research on the application of GBLM in hydrodynamic lubrication is almost blank. The theoretical lubrication research of liquid metal bearing can help to better understand its working principle, which is of great significance in product design. In this paper, the viscosity of GBLM at different temperatures was measured experimentally, and the viscosity of it at high temperature was obtained by fitting data; as the rotating speed of CTT is high and the GBLM's viscosity at high temperature is low, the turbulent lubrication model was established, and considering the special structure of $\mathrm{V}-\mathrm{g}$ B, the lubrication performance of liquid metal bearing was calculated and studied by coordinate transformation.

\section{Viscosity Measurement of Gallium Based Liquid Metal}

Viscosity is a basic property of fluid. It is the measurement of internal friction force between fluid molecules when the fluid is displaced by external force. It indicates the fluidity of fluid, generally varying with different fluids. The smaller the viscosity, the better the fluidity. As an important index to evaluate the quality of lubricants, the viscosity reflects the internal friction force of fluid and is directly related to the performance, wear degree, and service life of the bearing [18]. In addition, in the theoretical analysis of lubrication, it is necessary to know the viscosity of the lubricant, judge its flow form according to the working conditions, and then establish the theoretical lubrication model for analysis. Therefore, in order to carry out the theoretical analysis of the bearing lubrication performance, it is very important to measure the viscosity of gallium based liquid metal.

First, we prepared the GBLM, which in this paper was a gallium-indium-tin alloy with a mass ratio of 68.5:21.5:10. According to the configuration method described by Liu Jing's team in [19], the high-purity metal was selected according to the mass ratio and mixed in the container, which should be cleaned with deionized water. Then, the mixed raw materials were heated to a certain temperature until the metal melted, stirred slightly and cooled to room temperature to obtain GBLM. As GBLM is very easy to oxidize in air, gallium oxide will be produced, which will affect the viscosity and surface tension $[20,21]$. Therefore, impurity removal steps should be carried out. As is well known, $\mathrm{NaOH}$ can 
react with oxides to remove oxide impurities. We mixed a certain concentration of $\mathrm{NaOH}$ solution and the prepared GBLM alloy, both incompatible with each other proportionally; in addition, the density of the liquid metal was larger, so it will be layered after standing; then the LM after impurity removal can be obtained by taking the lower layer solution with a rubber tip dropper.

Considering that the capillary viscometer is easy to operate and the error is small, this paper used this method to measure the viscosity of GBLM. The experimental setup (Model SYD-265E kinematic viscometer, China, SANUO) is shown in Figure 1a. Given that the actual working temperature can reach $300{ }^{\circ} \mathrm{C}$, it was difficult for the test temperature to reach. Therefore, in order to obtain the working viscosity, first, the viscosity of GBLM at different temperatures was measured by changing the test temperature, and then the viscosity-temperature relationship was fitted by using the least squares method. The results are shown in Figure 1b, and the viscosity-temperature equation can be obtained as follows:

$$
\ln v=-0.004638 \times T-0.616012
$$

where $v$ is the kinematic viscosity and $T$ is the temperature in centigrade.

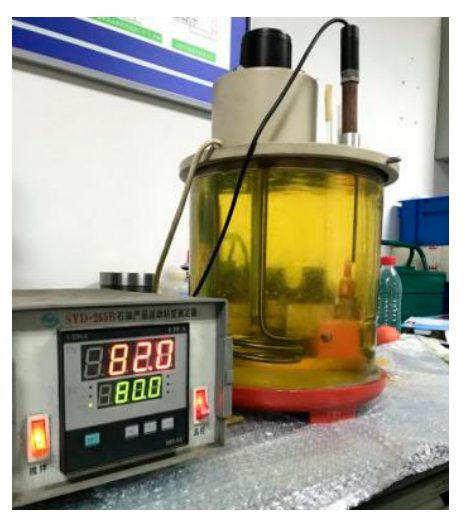

(a)

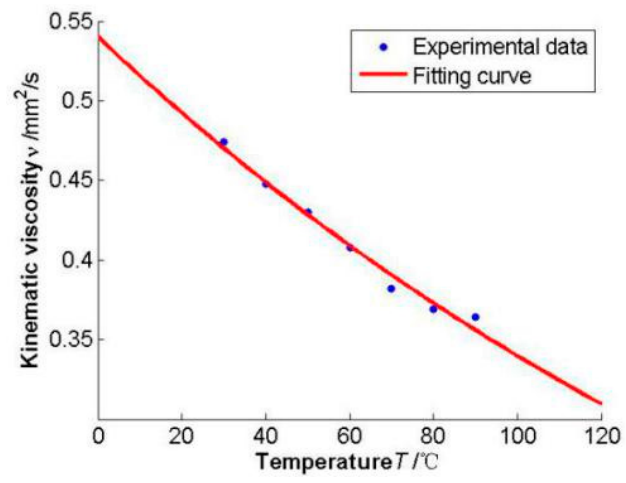

(b)

Figure 1. (a) The experimental setup for the capillary viscometer method. (b) The fitting curve of the viscosity-temperature relationship.

With Equation (1), the viscosity of GBLM under actual working conditions can be predicted: when the actual working temperature reached $300{ }^{\circ} \mathrm{C}$, the viscosity of GBLM was $0.134 \mathrm{~mm}^{2} / \mathrm{s}$, converted into a dynamic viscosity of $8.63 \times 10^{-4} \mathrm{~Pa} \cdot \mathrm{s}$.

By measuring the viscosity of GBLM, it was found that its viscosity was low under the actual working condition, and the rotating speed of the anode target in CTT was very high (up to about 10,000 r/min). Before the establishment of the theoretical model, the Reynolds number was calculated, which was about 2500. This exceeded the Reynolds number limit of laminar flow, which is 2000. Therefore, the turbulent model should be used in the theoretical analysis of lubrication.

\section{Numerical Model and Simulation}

The spiral groove bearing in the CT tube analyzed in this paper was a V-g B. Its essence is to machine a circle of V-groove on the surface of traditional smooth circular bearing. The structure is shown in Figure 2, in which, $\mathrm{h}$ is the oil film thickness; e is the eccentric distance; $\theta$ is the deflection angle; $b$ is the bearing width; $d$ is the bearing diameter; $\alpha$ is the included angle of herringbone groove; $\mathrm{Cg}$ is the trench depth; Lr the ridge width; and $\mathrm{Lg}$ is the groove width. 

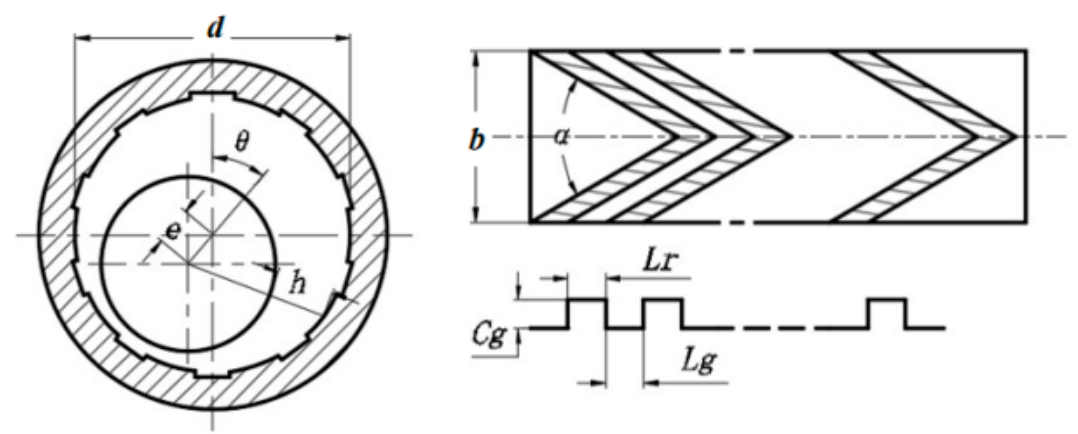

Figure 2. The structural diagram of V-g B.

\subsection{Establishment of Reynolds Equation for $V-G B$}

Considering that the $\mathrm{Ng}$-Pan turbulent model based on the law of the wall is the most widely used turbulent model. Meanwhile, its turbulent factor is linear, which makes the calculation more simple and suitable for light load hydrodynamic sliding bearing. Therefore, by choosing this model, the dimensionless Reynolds equation is as follows:

$$
\frac{\partial}{\partial \varphi}\left(\frac{H^{3}}{k_{\mathrm{x}}} \frac{\partial P}{\partial \varphi}\right)+\left(\frac{d}{b}\right)^{2} \frac{\partial}{\partial \lambda}\left(\frac{H^{3}}{k_{\mathrm{z}}} \frac{\partial P}{\partial \lambda}\right)=\frac{1}{4} \frac{\partial H}{\partial \varphi}
$$

where $k$ is the turbulent factor and the value is

$$
\left\{\begin{array}{c}
k_{\mathrm{x}}=12+0.0136(R e)^{0.9} \\
k_{\mathrm{z}}=12+0.0043(R e)^{0.98}
\end{array}\right.
$$

It can be seen from Figure 2 that the V-groove's structure is special because its trend is inclined in a straight line. The rectangular grid under the Cartesian coordinate system (CCS) will increase the difficulty in dealing with the flow discontinuity (i.e., at the groove step). Therefore, transforming coordinate, the calculation, and analysis were carried out in the oblique coordinate system (OCS) consistent with the groove direction. Figure 3 is a diagram of the coordinate transformation.

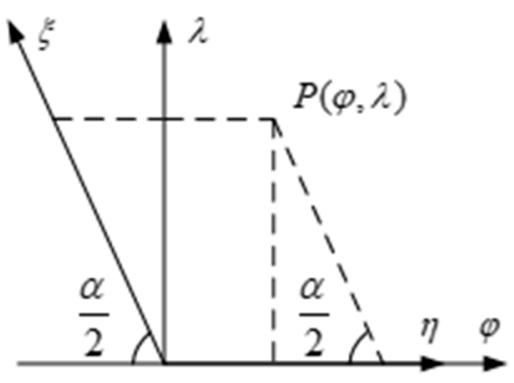

Figure 3. Diagram of coordinate transformation.

As shown in Figure 3, with the inclination angle of half of the included angle of the V-groove, the CCS was transformed into the OCS. The corresponding calculation relationship is

$$
\left\{\begin{array} { c } 
{ \varphi = \eta - \xi \operatorname { c o s } ( \frac { \alpha } { 2 } ) } \\
{ \lambda = \xi \operatorname { s i n } ( \frac { \alpha } { 2 } ) }
\end{array} \Leftrightarrow \left\{\begin{array}{c}
\eta=\varphi+\frac{\lambda}{\tan \left(\frac{\alpha}{2}\right)} \\
\xi=\frac{\lambda}{\sin \left(\frac{\alpha}{2}\right)}
\end{array}\right.\right.
$$


The expression of pressure partial derivative is obtained as followed:

$$
\left\{\begin{array}{c}
\frac{\partial P}{\partial \varphi}=\frac{\partial P}{\partial \eta} \frac{\partial \eta}{\partial \varphi}+\frac{\partial P}{\partial \xi} \frac{\partial \xi}{\partial \varphi}=\frac{\partial P}{\partial \eta} \\
\frac{\partial P}{\partial \xi}=\frac{\partial P}{\partial \eta} \frac{\partial \eta}{\partial \lambda}+\frac{\partial P}{\partial \xi} \frac{\partial \xi}{\partial \lambda}=\frac{1}{\tan \frac{\alpha}{2} \frac{\partial P}{\partial \eta}}+\frac{1}{\sin \frac{\alpha P}{2} \frac{\partial P}{\partial \xi}}
\end{array}\right.
$$

Then, bring Equation (5) into Equation (2), and the Reynolds equation in OCS is as follows:

$$
\begin{aligned}
& \frac{\partial}{\partial \eta}\left(\frac{H^{3}}{k_{\mathrm{x}}} \frac{\partial P}{\partial \eta}\right)+\frac{d^{2}}{b^{2} \tan ^{2}\left(\frac{\alpha}{2}\right)} \frac{\partial}{\partial \eta}\left(\frac{H^{3}}{k_{\mathrm{z}}} \frac{\partial P}{\partial \eta}\right)+\frac{d^{2}}{b^{2} \tan \left(\frac{\alpha}{2}\right) \sin \left(\frac{\alpha}{2}\right)} \frac{\partial}{\partial \eta}\left(\frac{H^{3}}{k_{\mathrm{z}}} \frac{\partial P}{\partial \xi}\right) \\
& \quad+\frac{d^{2}}{b^{2} \tan \left(\frac{\alpha}{2}\right) \sin \left(\frac{\alpha}{2}\right)} \frac{\partial}{\partial \xi}\left(\frac{H^{3}}{k_{\mathrm{z}}} \frac{\partial P}{\partial \eta}\right)+\frac{d^{2}}{b^{2} \sin ^{2}\left(\frac{\alpha}{2}\right)} \frac{\partial}{\partial \xi}\left(\frac{H^{3}}{k_{\mathrm{z}}} \frac{\partial P}{\partial \xi}\right)=\frac{1}{4} \frac{\partial H}{\partial \eta}
\end{aligned}
$$

At this time, the oil film thickness equation is:

ridge:

$$
H=1+\varepsilon \cos \varphi=1+\varepsilon \cos \left(\eta-\xi \cos \frac{\alpha}{2}-\theta\right)
$$

groove:

$$
H=1+\varepsilon \cos \varphi+\gamma_{g}=1+\varepsilon \cos \left(\eta-\xi \cos \frac{\alpha}{2}-\theta\right)+\gamma_{g}
$$

where $\gamma_{g}=\frac{c g}{c}$ is the groove depth ratio; $\varepsilon=\frac{e}{c}$ is the eccentricity; $c g$ is the groove depth; and $c$ is the radius gap.

\subsection{Equation Solving}

\subsubsection{The Discretization of Equations}

In general, the calculation in CCS can directly carry out differential processing of the partial derivative of the Reynolds equation. However, the equations calculated in OCS have cross partial derivatives, and the direct differential processing will make pressure values at non nodes. Therefore, the five-point difference method was not applicable here, and the local integration method was chosen to deal with this problem.

When the bearing is meshed along the direction of the V-groove and its boundary, there will appear continuous and discontinuous areas of oil film. As an example, the process of the local integration method can be explained by taking the node falling at the continuous area. The integral area is shown in Figure 4, recorded as marked area A.

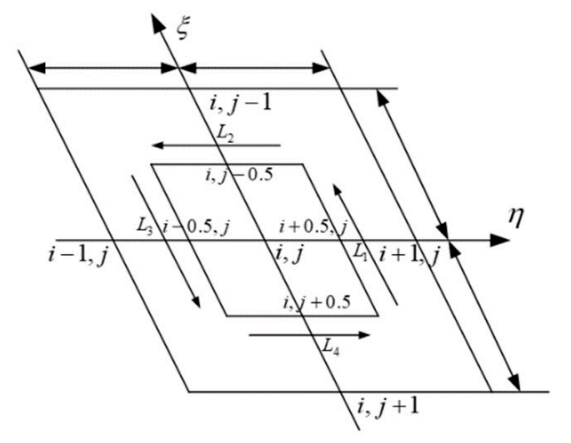

Figure 4. Diagram of continuous oil film integration area A.

Both sides of Equation (6) were conducted on a surface integral in the region shown below in Figure 5:

$$
\iint_{\mathrm{A}}\left[\frac{\partial}{\partial \varphi}\left(\frac{H^{3}}{k_{\mathrm{x}}} \frac{\partial P}{\partial \varphi}\right)+\left(\frac{d}{b}\right)^{2} \frac{\partial}{\partial \lambda}\left(\frac{H^{3}}{k_{\mathrm{z}}} \frac{\partial P}{\partial \lambda}\right)\right] \mathrm{d} \varphi \mathrm{d} \lambda=\frac{1}{4} \iint_{\mathrm{A}}\left(\frac{\partial H}{\partial \varphi}\right) \mathrm{d} \varphi \mathrm{d} \lambda
$$




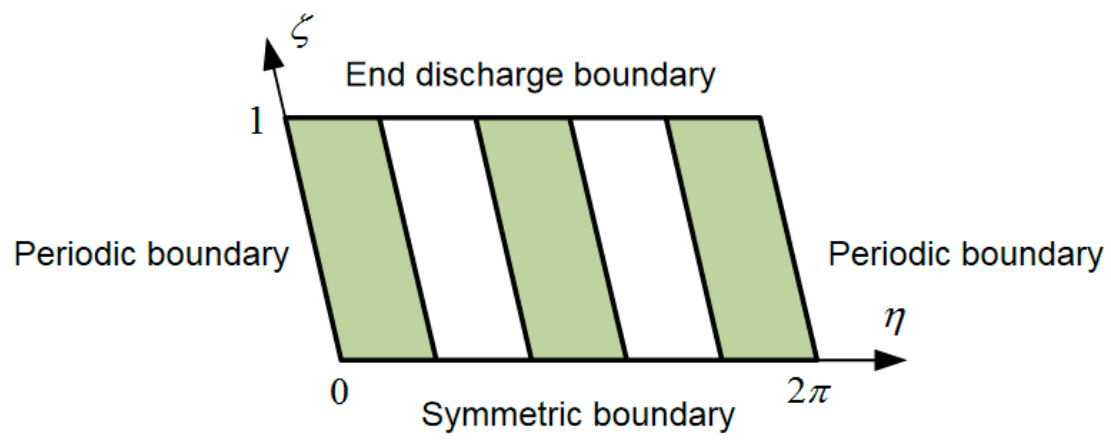

Figure 5. Diagram of the bearing boundary. follows:

Using the Green formula, the surface integral was changed into a curve integral as

$$
\int_{\mathrm{L}} \frac{H^{3}}{k_{\mathrm{x}}} \frac{\partial P}{\partial \varphi} \mathrm{d} \lambda-\left(\frac{d}{b}\right)^{2} \int_{\mathrm{L}} \frac{H^{3}}{k_{\mathrm{z}}} \frac{\partial P}{\partial \lambda} \mathrm{d} \varphi=\frac{1}{4} \int_{L} H \mathrm{~d} \lambda
$$

Taking Equations (4) and (5) into Equation (10), the integration was carried out according to the curve direction shown in Figure 5, and the five-point difference formula in oblique coordinate was obtained:

$$
P_{i, j}=\frac{A_{i, j} P_{i+1, j}+B_{i, j} P_{i-1, j}+C_{i, j} P_{i, j+1}+D_{i, j} P_{i, j-1}-F_{i, j}}{E_{i, j}}
$$

where

$$
\begin{gathered}
A_{i, j}=\frac{\Delta \xi}{\Delta \eta} \sin \frac{\alpha}{2} \frac{\mathrm{H}_{i+0.5, \mathrm{j}}^{3}}{k_{\mathrm{x}}}-\left(\frac{d}{b}\right)^{2}\left(\frac{-H_{i, j-0.5}^{3}+H_{i, j+0.5}^{3}}{2 k_{\mathrm{Z}} \tan \left(\frac{\alpha}{2}\right)}-\frac{\Delta \xi}{\Delta \eta} \frac{\cos \left(\frac{\alpha}{2}\right)}{\tan \left(\frac{\alpha}{2}\right)} \frac{H_{i+0.5, j}^{3}}{k_{\mathrm{z}}}\right) \\
B_{i, j}=\frac{\Delta \xi}{\Delta \eta} \sin \frac{\alpha}{2} \frac{\mathrm{H}_{i-0.5, j}^{3}}{k_{\mathrm{x}}}-\left(\frac{d}{b}\right)^{2}\left(\frac{H_{i, j-0.5}^{3}+H_{i, j+0.5}^{3}}{2 k_{\mathrm{Z}} \tan \left(\frac{\alpha}{2}\right)}-\frac{\Delta \xi}{\Delta \eta} \frac{\cos \left(\frac{\alpha}{2}\right)}{\tan \left(\frac{\alpha}{2}\right)} \frac{H_{i-0.5, j}^{3}}{k_{\mathrm{z}}}\right) \\
C_{i, j}=-\left(\frac{d}{b}\right)^{2}\left(-\frac{\Delta \eta}{\Delta \xi} \frac{1}{\sin \left(\frac{\alpha}{2}\right)} \frac{H_{i, j+0.5}^{3}}{k_{\mathrm{z}}}-\cos \frac{\alpha}{2} \frac{H_{i+0.5, j}^{3}+H_{i-0.5, j}^{3}}{2 k_{\mathrm{z}} \sin \left(\frac{\alpha}{2}\right)}\right) \\
D_{i, j}=-\left(\frac{d}{b}\right)^{2}\left(-\frac{\Delta \eta}{\Delta \xi} \frac{1}{\sin \left(\frac{\alpha}{2}\right)} \frac{H_{i, j-0.5}^{3}}{k_{\mathrm{z}}}-\cos \frac{\alpha}{2} \frac{H_{i+0.5, j}^{3}-H_{i-0.5, j}^{3}}{2 k_{\mathrm{z}} \sin \left(\frac{\alpha}{2}\right)}\right) \\
E_{i, j}=A_{i, j}+B_{i, j}+C_{i, j}+D_{i, j} \\
F_{i, j}=\frac{1}{4} \sin \left(\frac{\alpha}{2}\right) \Delta \xi\left(H_{i+0.5, j}-H_{i-0.5, j}\right)
\end{gathered}
$$

For the discontinuous areas of oil film (i.e., the nodes falling at the junction of ridge and groove), it is only necessary to locally integrate the left and right parts of Figure 4 , respectively. For the dynamic Reynolds equation, the small perturbation method is directly used to deal with this as the discrete form is similar to that of the static equation.

\subsubsection{Boundary Conditions}

Due to the symmetry of the V-groove structure, half of it was selected for research, as shown in Figure 5. 
The boundary conditions of static Reynolds equation for V-g B are as follows:

$$
\left\{\begin{array}{c}
\text { End discharge boundary }:\left.P\right|_{\xi= \pm \frac{1}{\sin \left(\frac{\alpha}{2}\right)}}=0 \\
\text { Periodic boundary }: P_{\eta=0}=P_{\eta=2 \pi} \\
\text { Reynolds boundary: } P_{\eta \geq \eta_{1}}=0 ;\left.\frac{\partial P}{\partial \eta}\right|_{\eta \geq \eta_{1}}=0
\end{array}\right.
$$

For the calculation of the symmetric boundary, just assign the pressure symmetrically.

The boundary condition of the dynamic Reynolds equation of V-g B is that the disturbance pressure was zero around the complete oil film area determined by the above static Reynolds boundary.

\subsubsection{Iterative Calculation}

The successive over-relaxation method (SOR method) is easy to apply with a wide application range, and its programming is simple. Therefore, the SOR method was used to solve the static and dynamic difference equations.

In order to judge whether the result of each iteration has reached enough accuracy, and then decide whether the iteration process can be stopped, the following convergence criteria were set here:

$$
\left(\sum_{j=2}^{m} \sum_{i=2}^{n}\left|P_{i, j}^{k}-P_{i, j}^{k-1}\right|\right) /\left(\sum_{j=2}^{m} \sum_{i=2}^{n}\left|P_{i, j}^{k}\right|\right) \leq \delta
$$

where the allowable relative error $\delta$ is generally about $10^{-3}$.

Figure 6 shows the iterative flow chart of the V-g B performance calculation.

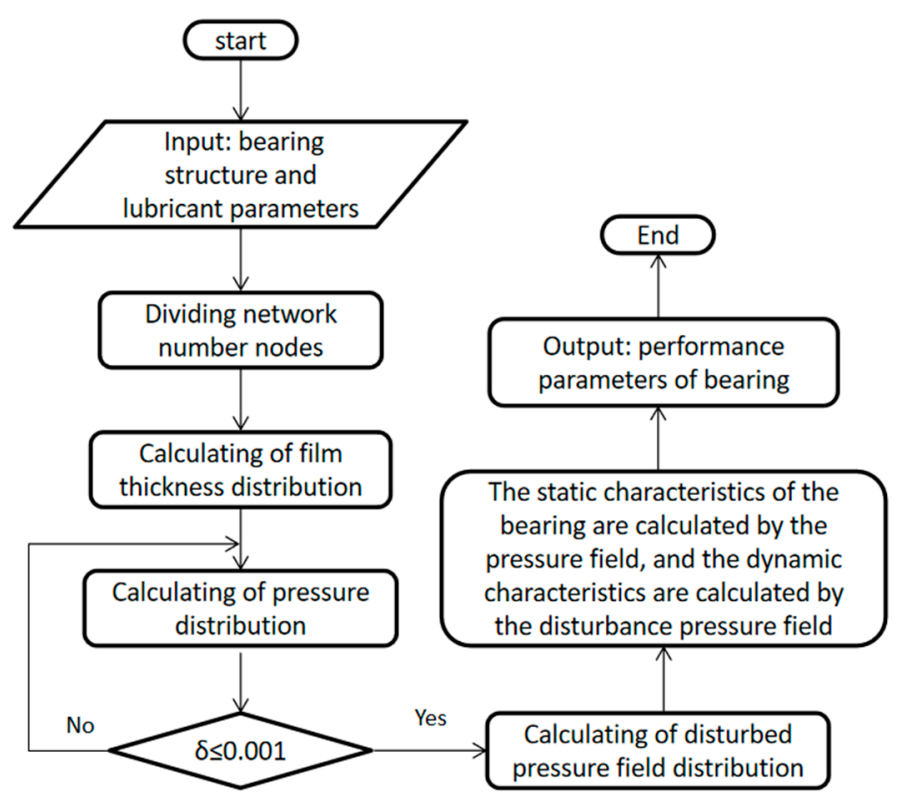

Figure 6. Flowchart of the numerical procedure.

\subsubsection{Performance Calculation}

In order to reduce the friction resistance of parts in operation and improve the bearing capacity of lubricating film, and then improve the stability of bearing performance, the pressure distribution, supporting force, friction resistance and stiffness of lubricating film should be calculated by using lubrication theory.

The following is the calculation formula for the bearing performance parameters: 
(1) Bearing capacity:

$$
\left\{\begin{array}{c}
\text { Total bearing capacity }: \bar{F}=\sqrt{{\overline{F_{\mathrm{x}}}}^{2}+{\overline{F_{\mathrm{y}}}}^{2}} \\
\text { Horizontal bearing capacity }: \overline{F_{\mathrm{x}}}=\int_{-1}^{1} \int_{0}^{2 \pi} P \sin \varphi \mathrm{d} \varphi \mathrm{d} \lambda \\
\text { Vertical bearing capacity }: \overline{F_{\mathrm{y}}}=\int_{-1}^{1} \int_{0}^{2 \pi} P \cos \varphi \mathrm{d} \varphi \mathrm{d} \lambda
\end{array}\right.
$$

(2) Deflection angle:

The deflection angle is solved by iteration. In the beginning, when the value of the bearing deflection angle is not known, an initial deflection angle should be given, and then the load angle can be calculated after calculating the oil film bearing capacity:

$$
\alpha_{\mathrm{F}}=\operatorname{arctg}\left(\frac{F_{\xi}}{F_{\eta}}\right)
$$

The deflection angle is corrected according to the calculated load angle:

$$
\theta=\theta_{0}-\alpha_{F}
$$

After the new deflection angle is obtained, the real offset angle can be obtained by returning to the program for iterative solution again until the deflection angle correction converges.

(3) Discharge:

$$
Q_{\mathrm{L}}=-4 c U b \int\left[\left(\left.\frac{r^{2}}{b^{2}} \frac{H^{3}}{k_{z}} \frac{\partial P}{\partial \lambda}\right|_{\lambda=1}\right)-\left(\left.\frac{r^{2}}{b^{2}} \frac{H^{3}}{k_{z}} \frac{\partial P}{\partial \lambda}\right|_{\lambda=-1}\right)\right] \mathrm{d} \varphi
$$

(4) Friction resistance, friction power consumption, and temperature rise:

Friction resistance:

$$
F_{\mathrm{t}}=\frac{\omega r^{2} \mu b}{2 c} \iint \frac{1}{H} \overline{\tau_{c}}-\frac{12 H}{k_{x}} \frac{\partial P}{\partial \varphi} \mathrm{d} \varphi \mathrm{d} \lambda
$$

where $\overline{\tau_{c}}$ is the Kutta shear stress; $\bar{\tau}_{c}=-1$ in laminar flow; and $\overline{\tau_{c}}$ is a function of Reynolds number in turbulent flow, which is obtained from the experimental data in [22]:

$$
\overline{\tau_{c}}=1+0.0012(R e)^{0.94}
$$

Friction power consumption:

$$
N_{t}=F_{t} \cdot U
$$

Temperature rise:

$$
\Delta T=\frac{F_{t} \cdot U}{Q_{L} \rho c_{v}}
$$

(5) Four dimensionless stiffness of bearing:

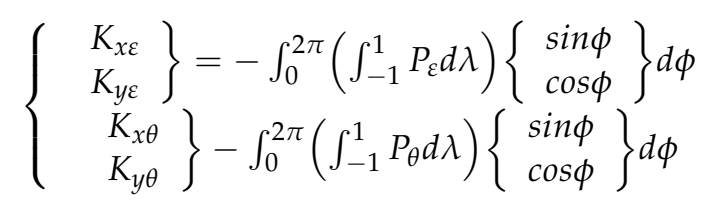


(6) Four dimensionless damping of bearing:

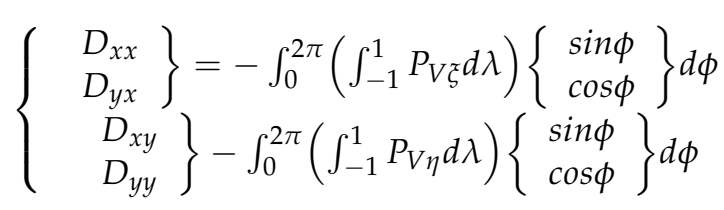

(7) Equivalent stiffness:

$$
K_{e q}=\left(K_{x x} D_{y y}+K_{y y} D_{x x}-K_{x y} D_{y x}-K_{y x} D_{x y}\right) /\left(D_{x x}+D_{y y}\right)
$$

(8) Limit eddy ratio square:

$$
\gamma_{s t}^{2}=\left[\left(K_{e q}-K_{x x}\right)\left(K_{e q}-K_{y y}\right)-K_{x y} K_{y x}\right] /\left(D_{x x} D_{y y}-D_{x y} D_{y x}\right)
$$

(9) Dimensionless critical mass:

$$
M=K_{e q} / \gamma_{s t}^{2}
$$

\subsection{Model Validation}

There has been validation between the above numerical model and references. One was the experimental study on the turbulent lubrication performance of the $360^{\circ}$ sliding bearing performed by Smith and Fuller [23], and another was the simulation result of bearing capacity in [24]. The results are shown in Figure 7.

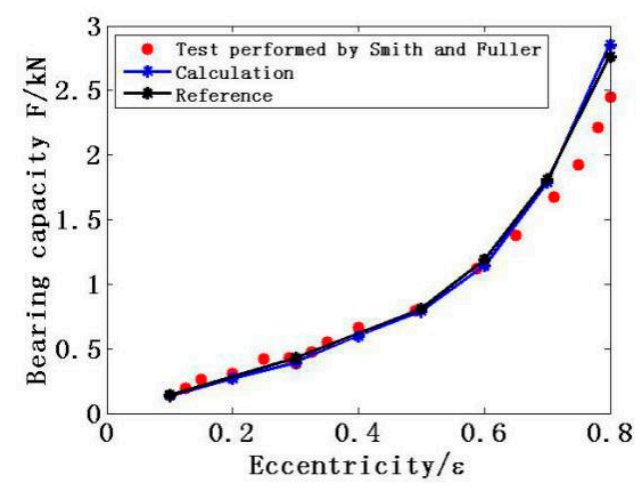

Figure 7. Verification of the bearing capacity of the circular bearing.

As shown in Figure 7, the numerical model was in good agreement with the literature and experimental results.

\section{Results and Discussion}

The parameters of V-g B and the lubricant used in the following analysis are listed in Tables 1 and 2.

Table 1. The parameters of V-g B.

\begin{tabular}{cc}
\hline Parameter & Numerical Value \\
\hline Bearing diameter d $/ \mathrm{mm}$ & 30 \\
Radius gap c/mm & 0.02 \\
Number of slots $\mathrm{n}$ & 10 \\
Groove-ridge ratio $\mathrm{r}_{\mathrm{g}}$ & 1 \\
Width-diameter ratio L/d & 1 \\
Helix angle $\alpha /{ }^{\circ}$ & 60 \\
Rotation rate $\mathrm{N} /\left(\mathrm{r} \cdot \mathrm{min}^{-1}\right)$ & 10,800 \\
Groove depth $\mathrm{cg} / \mathrm{mm}$ & 0.02 \\
\hline
\end{tabular}


Table 2. The parameters of the lubricant.

\begin{tabular}{cc}
\hline Parameter & Numerical Value \\
\hline Density & $6.44 \mathrm{~g} / \mathrm{cm}^{3}$ \\
Viscosity & $1.8 \times 10^{-3} \mathrm{~Pa} \cdot \mathrm{s}$ \\
Specific heat capacity & $365.6 \mathrm{~J} / \mathrm{kg} \cdot{ }^{\circ} \mathrm{C}$ \\
\hline
\end{tabular}

\subsection{Comparison of Cartesian and Oblique Coordinates}

With the eccentricity of 0.4 , Figure 8 shows the oil film thickness and pressure distributions of liquid metal bearing, where (a) and (b) are calculated in CCS, and (c) and (d) are calculated in OCS.

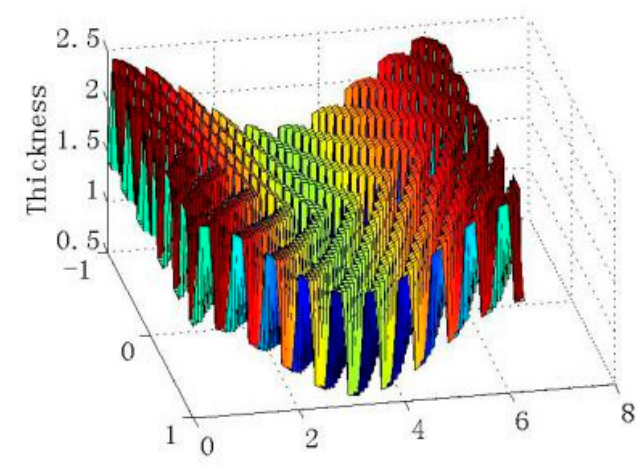

(a)

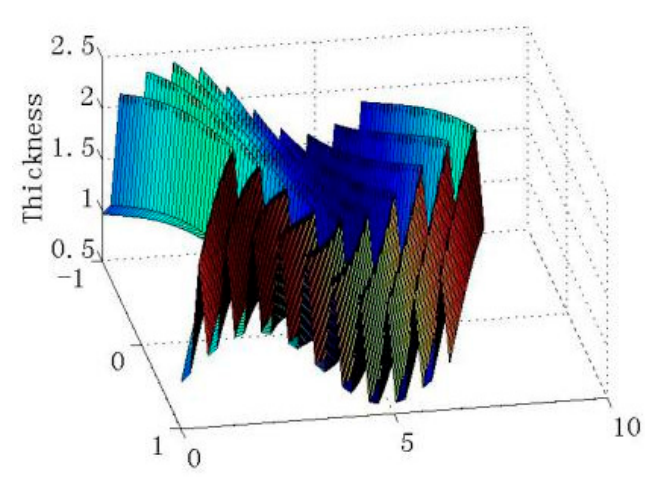

(c)

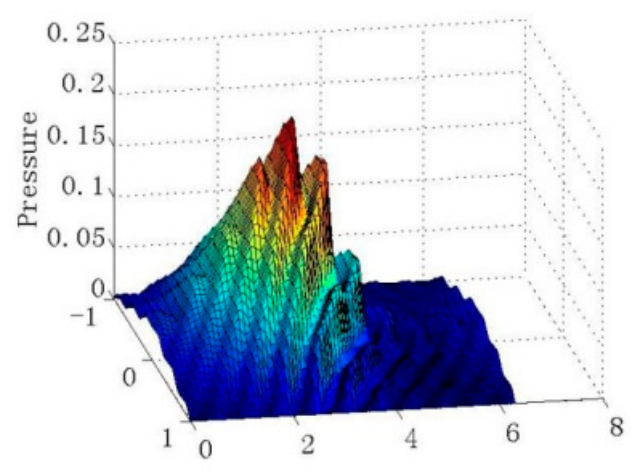

(b)

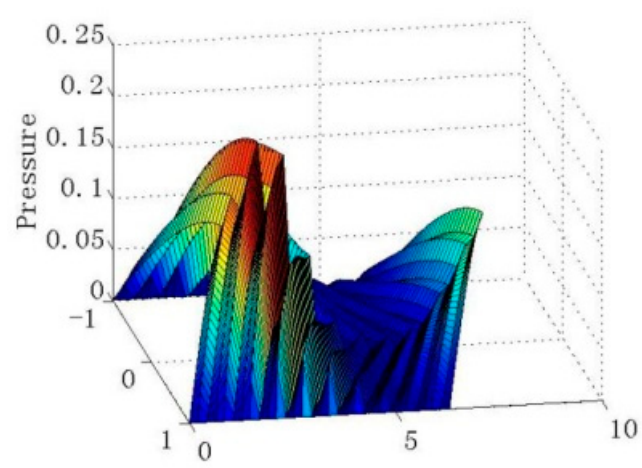

(d)

Figure 8. Oil film thickness and pressure distributions in different coordinate systems: $(\mathbf{a}, \mathbf{b})$ are calculated in CCS; (c,d) are calculated in OCS.

As shown in Figure 8, it can be seen that, in different coordinates, the film thickness and pressure distributions were consistent, respectively, and the pressure value reached the maximum near the minimum film thickness. Compared with the distribution calculated in CCS, the film thickness and pressure distribution calculated in OCS were smoother, and the direction of iteration was more consistent with the shape boundary of the V-shaped groove. It was more consistent with the supporting principle of $\mathrm{V}$-shaped spiral groove bearing as well as closer to the flow direction of the lubricating medium.

The film thickness distribution of the circumferential was similar to that of the circular bearing. However, as the V-groove was uniformly distributed on the bearing surface, it presented a step distribution. Due to the existence of wedge effect, the pressure distribution in the circumferential direction was similar to that of the circular bearing.

In this paper, the spiral groove was set on the shaft and the corresponding shaft sleeve cavity was smooth, the shaft rotated at a certain speed, and the liquid on one side of the 
sleeve was pumped. This phenomenon is called the pumping pressure effect. Therefore, because of the existence of the V-groove's pumping pressure effect, the pressure at different positions in the circumferential direction increases rapidly at the tip of the V-groove center, forming a local peak. Moreover, as a result of the existence of both effects, almost every V-groove corresponded to a local peak.

\subsection{Comparative Analysis of Small Eccentricity and Large Eccentricity}

Setting the eccentricity to 0.1 and 0.6 , respectively, the bearing pressure distribution was calculated by using the model described in the previous section.

Figure 9 shows the calculated bearing pressure distribution, where (a) is calculated with the eccentricity of 0.1 , and (b) is calculated with the eccentricity of 0.6 .

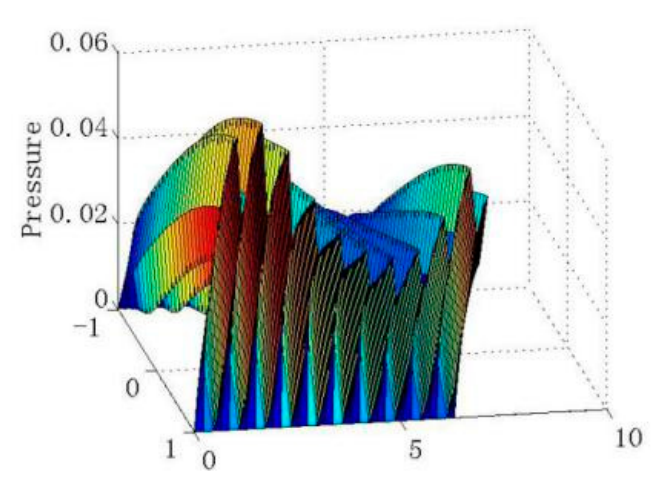

(a)

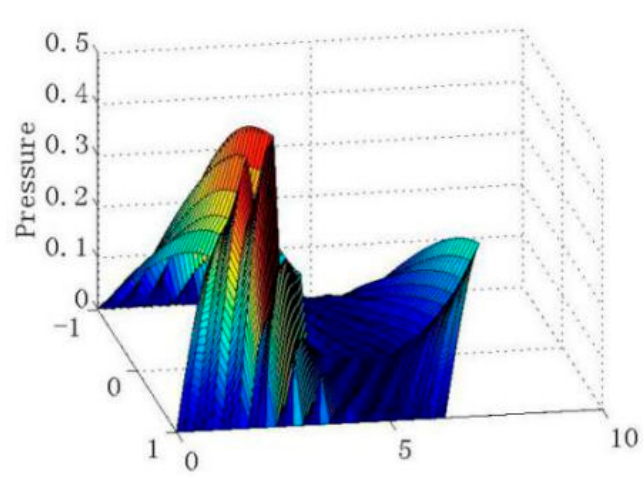

(b)

Figure 9. Pressure distributions under different eccentricities: (a) Dimensionless pressure distributable 0.1; (b) Dimensionless pressure distribution calculated with the eccentricity of 0.6 .

From the analysis of Figure 9, the pressure tends to be gentle along the circumference with the small eccentricity, and the pressure peak value is prominent and changes violently when the eccentricity is large. In the case of small eccentricity, the pressure is mainly provided by the V-groove's pumping pressure effect and relatively scattered, which is mainly distributed in the groove rather than at the ridge; meanwhile, each V-groove corresponds to a local pressure peak point with a small value. In the case of large eccentricity, the overall wedge-shaped effect of bearing is more obvious, the pressure distribution is more concentrated with a larger peak value, which is mainly distributed in the ridge, while at the same time, the pump pressure effect is evident near the minimum film thickness.

Regardless of eccentricity, compared with ordinary smooth bearing, V-g B had a more dispersed pressure distribution with a wider range, more peak points, and more stable pressure change.

\subsection{Static and Dynamic Performance Analysis of Bearing}

The static and dynamic performances of hydrodynamic V-g B was analyzed by changing eccentricity from 0.1 to 0.9 while other parameters remained unchanged.

Figure 10 shows the curve of the static and dynamic performance parameters of V-g B with eccentricity. 


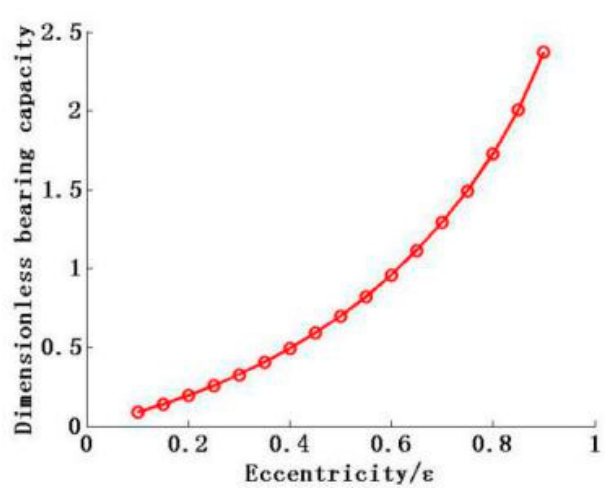

(a)

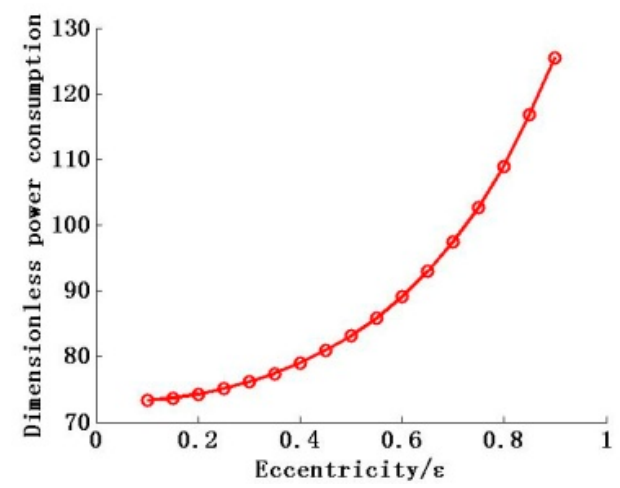

(c)

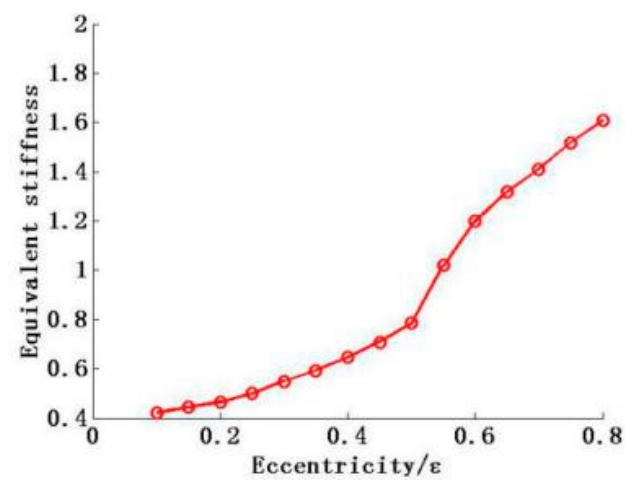

(e)

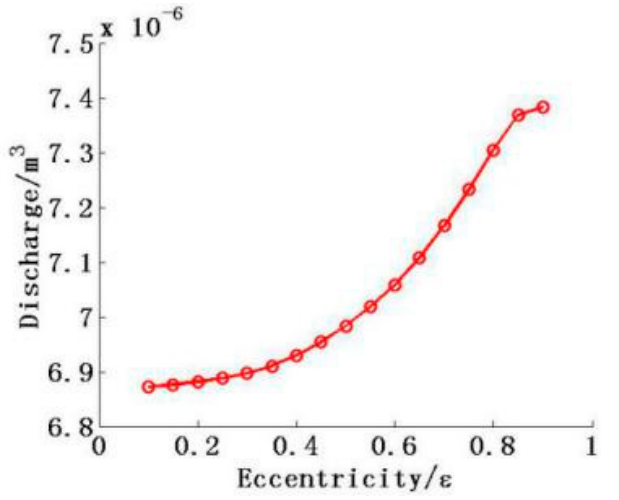

(b)

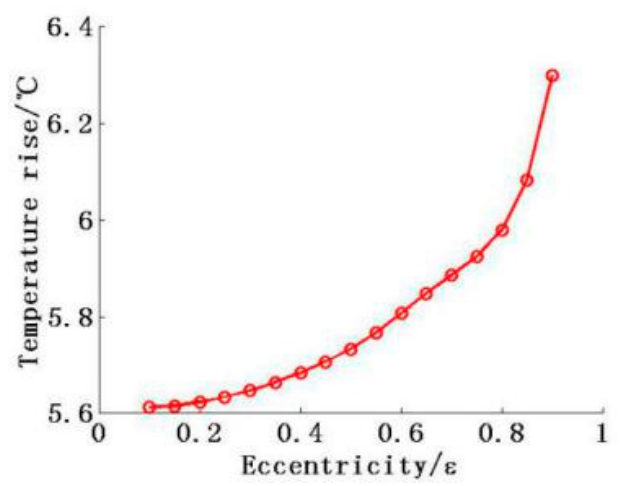

(d)

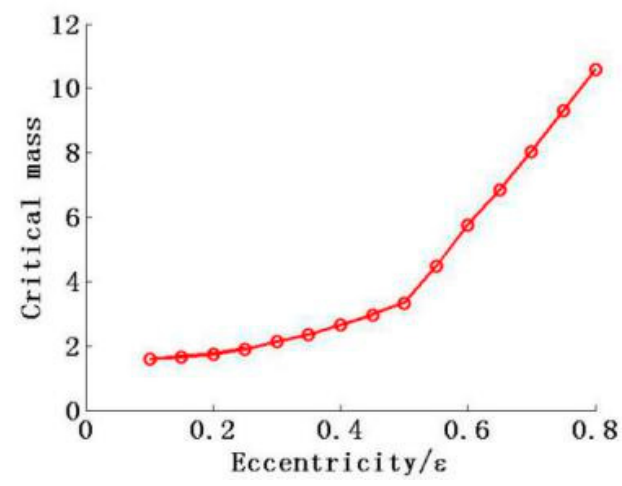

(f)

Figure 10. Curve of the performance parameters of V-g B with eccentricity: (a) Dimensionless bearing capacity; (b) Discharge; (c) Dimensionless power consumption; (d) Temperature rise; (e) Dimensionless equivalent stiffness; (f) Dimensionless critical mass.

Based on the analysis, it can be found that with the increase in eccentricity, the clearance between the bearing and bearing bush decreases, leading to the increase of the wedge effect, then the oil film pressure increases as well as the bearing capacity. At the same time, the discharge increases linearly with the increase of eccentricity. When the eccentricity increased to about 0.6, the oil film became thinner, causing a significant rise in the shear force, then the friction power consumption increased rapidly. Therefore, due to the combined influence of friction power consumption and discharge, the temperature rise increases with the trend of eccentricity, which rose rapidly when the eccentricity climbed to 0.6 , and the total temperature rise was within $6.4^{\circ} \mathrm{C}$. Simultaneously, the stiffness and critical mass kept enhancing with the increase of eccentricity. 


\section{Conclusions}

In this paper, the viscosity of G-I-T LM was measured, and the relationship curve between viscosity and temperature was fitted. After considering the influence of turbulence, the film thickness and pressure distribution of the bearing calculated in CCS and OCS were analyzed and compared by using the $\mathrm{Ng}$-Pan turbulent model; the difference of bearing pressure distribution under small and large eccentricity was studied, respectively, and the static and dynamic performances of GBLM lubricated V-g B such as bearing capacity, discharge, friction power consumption, temperature rise, stiffness and critical mass, with change of eccentricity were analyzed. The conclusions are as follows:

1. The viscosity of G-I-T LM will decrease with the increase of temperature, so under the working condition of high speed and high temperature, the flow form of G-I-T LM is turbulent flow.

2. The spiral groove bearing's carrying capacity is provided by the pump pressure effect and wedge effect together: in the case of small eccentricity, the pump pressure effect of the herringbone groove mainly provides pressure, and the overall wedge effect of bearing is more obvious under large eccentricity.

3. The static and dynamic performance parameters of bearing increase with the rise in eccentricity.

Author Contributions: Methodology, M.X.; investigation, M.X.; writing-original draft, M.X.; supervision, W.C.; writing-review and editing, W.C. All authors have read and agreed to the published version of the manuscript.

Funding: This research was funded by the National Science and Technology Major Project of China (grant number 2017YFC0111500).

Institutional Review Board Statement: Not applicable.

Informed Consent Statement: Not applicable.

Data Availability Statement: The data presented in this study are available on request from the corresponding author.

Acknowledgments: We acknowledge the support of the National Science and Technology Major Project of China and $\mathrm{Xi}^{\prime}$ an Jiaotong University.

Conflicts of Interest: The authors declare that there is no conflict of interests regarding the publication of this article.

\section{References}

1. Shi, L.; Zhang, F.; Wang, R.; Wang, Y. Domestic and foreign situation and development trend of medical CT X-ray tubes. Vac. Electron. 2018, 2, 61-68.

2. Gerkema, J.; Pelzer, J.B. Metal-Lubricated Helical-Groove Bearing Comprising an Anti-Wetting Layer. U.S. Patent 4,614,445, 15 May 1985.

3. Bittl, H. X-ray Tube with Liquid-Metal Fluid Bearing. U.S. Patent 7,050,541, 21 April 2004.

4. Pinera, A.; Pelfrey, P.C. Hydrodynamic Liquid Metal Bearing. U.S. Patent 7,896,550, 2 May 2007.

5. Turaga, R.; Parampil, G. Bearing Mechanism and X-ray Tube. U.S. Patent 5,483,570, 5 July 2006.

6. Li, Y.; Yang, P.; Chen, W. A study of bearing design for liquid metal lubricated spiral grooves under influences of turbulence and slip. J. Xi'an Jiaotong Univ. 2019, 53, 15-23.

7. Cheng, J.; Yu, Y.; Zhu, S.; Ye, Q.; Liu, W. Lubrication characteristics of multifunctional lliquid-state metal materials under different sliding-pairs. Tribology 2017, 37, 435-441.

8. Hughes, W.F. Magnetohydrodynamic lubrucation and application to liquid metals. Ind. Lubr. Tribol. 1963, 15, 125-133. [CrossRef]

9. Burton, R.G.; Burton, R.A. Gallium alloy as lubricant for high current density brushes. IEEE Trans. Compon. Hybrids Manuf. Technol. 1988, 11, 112-115. [CrossRef]

10. Gerkema, J. Gallium-based liquid-metal full-film lubricated journal bearings. ASLE Trans. 1985, 28, 47-53. [CrossRef]

11. Kezik, V.Y.; Kalinichenko, A.S.; Kalinitchenko, V.A. The application of gallium as a liquid metal lubricant. Z. Für Met. 2003, 94, 81-90. [CrossRef]

12. Li, H.J.; Tian, P.Y.; Lu, H.Y.; Jia, W.; Du, H.; Zhang, X.; Li, Q.; Tian, Y. State-of-the-art of extreme pressure lubrication realized with the high thermal diffusivity of liquid metal. ACS Appl. Mater. Interfaces 2017, 9, 5638-5644. [CrossRef] [PubMed] 
13. Guo, J.; Cheng, J.; Tan, H.; Zhu, S.; Qiao, Z.; Yang, J.; Liu, W. Ga-based liquid metal: A novel current-carrying lubricant. Tribol. Int. 2019, 135, 457-462. [CrossRef]

14. Cheng, J.; Yu, Y.; Guo, J.; Wang, S.; Zhu, S.; Qian, Y.; Yang, J.; Liu, W. Ga-based liquid metal with good self-lubricity and high load-carrying capacity. Tribol. Int. 2019, 129, 1-4. [CrossRef]

15. Cheng, J.; Zhu, S.; Tan, H.; Yu, Y.; Yang, J.; Liu, W. Lead-bismuth liquid metal: Lubrication behaviors. Wear 2019, 430, 94-99. [CrossRef]

16. Liu, W.; Xu, J.; Feng, D.; Wang, X. The research status and prospect of synthetic lubricating oils. Tribology 2013, 33, 91-104.

17. Wang, J.; Chen, F.; Chen, L.; Chen, Y. A review on groove bearings. Lubr. Eng. 2017, 42, 1-7.

18. Liu, Y.; Geng, H.R.; Sun, M.H.; Cui, H.W. Present status and future development of the research on liquid metal viscosity. Foundry 2000, 49, 875-878.

19. Zheng, Y.; He, Z.; Gao, Y.; Liu, J. Direct desktop printed-circuits-on-paper flexible electronics. Sci. Rep. 2013, 3, 1786. [CrossRef]

20. Qin, Q.; Liu, Y.; Wang, Y.; Runwei, L. Recent progress of methods for fabricating flexible conductive wires based on liquid metals. Electron. Compon. Mater. 2017, 36, 1-8.

21. Regan, M.J.; Tostmann, H.; Pershan, P.S.; Magnussen, O.M.; DiMasi, E.; Ocko, B.M.; Deutsch, M. X-ray study of the oxidation of liquid-gallium surfaces. Phys. Rev. B 1997, 55, 10786-10790. [CrossRef]

22. Chun, S.M. A parametric study on bubbly lubrication of high-speed journal bearings. Tribol. Int. 2002, 35, 1-13. [CrossRef]

23. Smith, M.I.; Fuller, D.D. Journal-bearing operation at super-laminar speeds. Trans. ASME 1956, 78, 469-474.

24. Wang, X.; Su, X.; Zhang, Z. Study on the performance of journal bearing Based in turbulent regime with the combined Reynolds stress model. J. Mech. Eng. 2003, 39, 85-89. [CrossRef] 